\title{
Trajectory changes are susceptible to 2 change blindness manipulations
}

4 Authors: Matt Jaquiery ${ }^{1 *}$, Nora Andermane ${ }^{2}$, Ron Chrisley ${ }^{3}$

5 Affiliation: ${ }^{1}$ Department of Experimental Psychology, University of Oxford, Oxford, UK.

$6 \quad 2$ Department of Psychology, University of Sussex, Falmer, Brighton, UK

$7{ }^{3}$ Department of Informatics, Centre for Cognitive Science (COGS), Sackler Centre for

8 Consciousness Science, University of Sussex, Falmer, Brighton, UK

$9 \quad{ }^{*}$ Corresponding author

Email: matt.jaquiery@psy.ox.ac.uk

Abstract

People routinely fail to notice that things have changed in a visual scene if they do not perceive the changes in the process of occurring, a phenomenon known as 'change blindness' $(1,2)$. The majority of lab-based change blindness studies use static stimuli and require participants to identify simple changes such as alterations in stimulus orientation or scene composition.

This study uses a 'flicker' paradigm adapted for dynamic stimuli which allowed for both simple orientation changes and more complex trajectory changes. Participants were required to identify a moving rectangle which underwent one of these changes against a background of moving rectangles which did not. The results demonstrated that participants' ability to correctly identify the target deteriorated with the presence of a visual mask and a larger number of distractor objects, consistent with findings in previous change blindness work. with dynamic stimuli, and that changes to predictable trajectories are detected or missed in the similar way as orientation changes. 


\section{Introduction}

26 People routinely fail to notice that objects have changed in a visual scene if they do not perceive the changes in the process of occurring, a phenomenon known as 'change blindness' $(1,2)$. The majority of lab-based change blindness studies use static stimuli and require participants to identify simple changes such as alterations in stimulus orientation or scene composition (3-5), though others use more complex and realistic environments, especially driving simulators (6-8). This study examines whether changes to dynamic properties are detected or missed in the same way as changes to static properties.

Changes to static properties (e.g. the presence of a stimulus, or its orientation) are most readily detected when the transients (moment-to-moment variations) accompanying a change prompt an explicit comparison between a stored representation of a stimulus and its current presentation (9-11). Change blindness frequently occurs when this process is disrupted. If the representation of a stimulus includes information on dynamic properties (e.g. the trajectory along which a stimulus is travelling), change blindness would be expected to occur for changes to dynamic as well as static stimulus properties. Common methodologies for inducing change blindness prevent transient registration, typically by masking (12) or eliminating transients $(1,13,14)$. In addition to masking transients, exhaustion of working memory capacity is required to produce change blindness effects reliably (15), with the contents of working memory exhibiting resistance to change blindness $(2,16)$, a phenomenon which is stable enough to allow change blindness task performance to act as a guide to working memory contents in attentional bias studies (17-19).

Change blindness as deployed in the study of other phenomena (e.g. attentional biases) is reasonably well understood, but a gap exists between these structured laboratory experiments and the more sophisticated simulator-based and natural-world experiments $(1,20,21)$. The use of dynamic paradigms such as video footage $(22,23)$ or programmed displays $(24)$ is required 
50 for a detailed examination of the processes underpinning change blindness within busy,

51 continually changing visual fields like those typical of everyday life (25).

52 Two key areas of enquiry are addressable with the use of dynamic stimuli: the nature of 53 competition between transients in exogenous orienting ('grabbing') of attention; and the existence of an ability to make discriminations between patterns of transients. The first of these areas is to some extent already established by the existence of change blindness paradigms in which attention is directed away from transients by the application of 'mudsplashes' or similar distractors (12): the more prominent transients accompanying the mudsplashes outcompete those accompanying the change in the target stimulus leading to observable change blindness. The second area of enquiry, discrimination between patterns of transients, is only available in dynamic scenes where changes are already occurring, and requires the detection not of specific transients but of a change in the pattern of those transients. A change in a pattern of transients signifies a change in the way in which a change is occurring (e.g. an acceleration or a change in the direction of movement).

Change blindness to changes in trajectory has been demonstrated in macaque monkeys in a study which used a change in the flow direction of dots within a field, with distractor fields in which the flow direction remained constant also visible (26). The present experiment establishes a related finding in humans, namely that trajectory changes can drive attentional mechanisms in the same manner as orientation changes, demonstrating detection of and blindness to changes in an object's dynamic properties where the alterations to the patterns 


\section{Materials \& Methods}

\section{Study structure}

73 The results presented below comprise an online study and a lab-based replication. Both 74 studies used the same materials and methods; however, the experimental environment and presentation was standardized for the lab cohort.

\section{Participants}

Participants ( $N$ online $=42, N$ lab $=16)$ were recruited to the study. Online participants were recruited via university mailing lists and social networking websites, while lab participants were recruited via the mailing lists and word-of-mouth. Prior to beginning the task participants were informed that no personally identifying information would be recorded, that participation was voluntary and could be halted at any time, and that they would be identified by means of temporary browser cookies. The experiment was administered online and demographic data such as age, education, and gender identity were not collected. It is likely the majority of the online cohort were undergraduates. The lab cohort were undergraduate and postgraduate students at the University of Sussex.

Target sample size for the online cohort was determined by precedent. Similar change blindness paradigms administered under laboratory conditions have used sample sizes in the $10-20$ range $(1,2,5,12,17,18,27)$. Given the reduction in precision accompanying the novel online administration in the present study, the previous range was doubled, resulting in a target sample size of 20-40. Active recruitment lasted two weeks, although participants were free to enter the study until the pre-established one-month data collection window had closed. Power analysis was used to determine the number of participants required for the lab-based study to produce $95 \%$ power for detecting the key interaction between masking and load as demonstrated in the online data. 
95

96

98

99

100

101

102

103

104

105

106

107

108

109

110

111

112

113

114

\section{Ethics}

This research was conducted in accordance with the ethics procedures of the University of Sussex School of Life Sciences, and approved by the University of Sussex Life Sciences School Research Ethics Officer on behalf of the Cluster-based Research Ethics Committee Ethical Review Application ID (ER/MJ261/1). After being briefed about the content and nature of the task the participants were required to signal consent by following a hyperlink to the task page. Participants were informed that participation was voluntary and that they were free to stop at any time. Participation was unpaid, but the lab cohort were entitled to receive a small amount of course credit.

\section{Task}

The participants completed the task by visiting a webpage which used JavaScript to deliver a dynamic version of a 'flicker' paradigm (2). In a typical flicker paradigm, as in most change blindness paradigms, the task is to detect changes between two stimuli. In the flicker paradigm the participant is shown one stimulus, then the other, repeatedly. Importantly, the stimuli presentations are separated by a brief presentation of a blank screen (the 'mask'). If the stimuli are switched without a mask the parts of the scene that are different produce visual transients, attracting attention to the location of the change. If, however, the switch is accompanied by the mask, the offset of the first stimulus and the onset of the second both produce transients throughout the visual scene, resulting in no net increase in attention to the location of the change.

The task page presented participants with a $700 \times 700$ pixel working area. The initial stimulus consisted of a number of 50x25 pixel rectangles with randomly selected colours moved at 150 pixels/second along a straight-line trajectory (Fig 1a). On low load trials there were 2 rectangles; on high load trials there were 6 . The direction of movement for each rectangle was determined randomly, subject to the constraints that: a) the rectangle could travel along the selected trajectory for the duration of the trial without leaving the working area; and b) there 
existed at least one possible altered trajectory which would not leave the working area. The

122 direction of movement bore no relation to the orientation of the rectangle. The alternate

123 stimulus matched the initial stimulus except that one of the rectangles had either its orientation

124 or its trajectory altered by $\pm 90^{\circ}$ (the 'change type' manipulation). Each stimulus was displayed

125 for $700 \mathrm{~ms}$ (a discussion of the precision of this timing is included below: Error! Reference source not found.). The alternate stimulus was either presented immediately after the initial stimulus' 700ms display duration ('unmasked' condition) or after a 200ms mask ('masked' condition). During the mask the rectangles were rendered invisible, resulting in a plain white background. Crucially, all rectangles vanished and reappeared at the same time. Once the alternate stimulus had been displayed for $700 \mathrm{~ms}$ the trial was restarted (Fig $1 \mathrm{~b}$ ), either immediately (unmasked condition) or following a second $200 \mathrm{~ms}$ mask. Trials continued until the participant provided a response. A demonstration video showing 10 trials can be found at (doi:10.6084/m9.figshare.5044894).

Fig 1. The task display and presentation procedure. a) Screenshot of the $700 \times 700$ pixel working area of the screen showing rectangles of random colours (selected from an approved region of colour-space) which moved in straight lines through the working area. After $700 \mathrm{~ms}$ one of the rectangles would alter either its orientation or its trajectory by $90^{\circ}$ either clockwise or anticlockwise. The task was to identify which of the rectangles had undergone this change. In the low load condition only two rectangles were presented; the high load condition presented six rectangles as shown. b) Flicker paradigm procedure. The initial scene (A) was displayed for $700 \mathrm{~ms}$, then a mask was put up for $200 \mathrm{~ms}$ (masked condition) or $0 \mathrm{~ms}$ (unmasked condition) before the initial scene was replaced with the altered scene ( $\left.A^{\prime}\right)$. The altered scene was displayed for $700 \mathrm{~ms}$ and then masked and reverted to the initial scene. The process was repeated until the participant generated a response.

146 Research on multiple object tracking (28) has shown that, although object tracking does not 147 interact with memory processes (29) (though see (30) for a dissenting view), tracked objects 
148 are to some extent resistant to change blindness $(31,32)$.; tracking target selection is typically

149 exogenous and based on colour and spatial location (33), As both of these were randomised

150 in all trials, there was no systematic relationship between the likelihood of an object being

151 tracked and its being the target object for that trial. Therefore, the dynamic paradigm did not

152 undermine the validity of the change blindness.

153 Participants were asked to press the spacebar as soon as they had identified the altered rectangle. Pressing spacebar halted the movement of the rectangles and correct identification was checked by requiring the participants to click the rectangle which had changed. Participants were given the opportunity to practice the task until they were satisfied with their performance, and were provided with feedback as to their accuracy during the practice.

Each trial had one of eight possible types, defined by its specific arrangement of three different binary variables: whether a mask was present or not; whether scene load was low (2 rectangles) or high (6 rectangles); and whether the target rectangle was changed in orientation or trajectory. Experimental condition was selected randomly at the beginning of each trial. The probability of low scene load was $30 \%$, chosen because more errors were expected under high load. Mask presence (present or absent) and change type (orientation or trajectory change) were equally likely for both options (50\%). The outcome measure was time elapsed between the beginning of the first presentation of the altered state and the moment the spacebar was depressed.

After every 10 trials participants were provided with statistics showing their accuracy and average speed over the last 10 trials, as well as their averages for all trials thus far completed. This provided a sense of progress for participants, and encouraged them to focus on fast and accurate responses. At the end of all 50 trials participants were shown their average accuracy and speed, as well as the average accuracy and speed for all participants combined.

172 Participants were invited to complete as many trials as they wished, though the provision of

173 full feedback after 50 trials was intended to incentivise the completion of at least 50 trials per 
174 participant. The overall number of trials, and the number of each type of trial seen by each

175 participant was subject to some variation since some participants completed more trials than

176 others and trial type was selected randomly at the beginning of each trial.

177 The lab participants completed 100 trials, and were given the information about their 178 performance relative to others only after they have completed these 100 trials.

179 The task application was coded in HTML and JavaScript with the aid of the CraftyJS 180 JavaScript game engine library version 0.7 .0 (34). Results were sent using AJAX 181 (Asynchronous JavaScript And XML) to a PHP script which stored them in a MySQL database and returned statistics to the participant when required. The JavaScript application was checked for compatibility with and parity between recent versions of the most common browsers (Microsoft Internet Explorer, Mozilla Firefox, Apple's Safari, and Google Chrome). Statistical analysis of data was performed using $R(35)$ and its tidyverse package (36). Initial analyses, reported in supplementary material, were performed using IBM SPSS Statistics package (version 22.0.0).

\section{Variation within the online cohort}

189 The use of web-based psychological experiments is an increasingly popular approach to acquiring data which offers a number of trade-offs compared to laboratory experiments (3739). Those advantages which are most salient to this project are the savings in time, money, and equipment, as well as the added convenience for participants who would have otherwise had to attend a laboratory session. Relevant disadvantages are primarily the result of nonstandardised equipment (including screen brightness, size, and resolution) and environments (including noise levels and distractions) resulting in a slightly different experience of the experiment for each participant.

197 The paradigm was implemented with JavaScript. JavaScript's control (used for stimulus 198 timings) is less precise than other frequently-used languages (40). There was thus unsystematic variation in stimulus and mask durations of $\pm 10 \mathrm{~ms}$, as well as some variation in 
200 the magnitude of this variation between browsers (on the order of $\pm 5 \mathrm{~ms}$ ). The unsystematic

201 variation constitutes noise, a factor addressed in the sample size (Participants). Variation on

202 the basis of browser is handled statistically as a component of inter-participant variation

203 (Error! Reference source not found.).

204 The retinal speed of stimuli was subject to variation between participants on the basis of screen size and viewing distance (which were not controlled). This variation is handled statistically as inter-participant variation (Error! Reference source not found.). A more pressing concern is the possibility that the experimental conditions may have been differentially affected by differences in stimulus retinal speed given that one of the conditions (trajectory change) was implemented through a change in the motion of the stimulus. This concern would be apt for manipulations in which the speed of the stimulus was altered (e.g. examining change detection for acceleration), but does not apply when, as here, the speed is kept constant while the direction of motion is altered.

213 The trade-off between these various factors was considered acceptable given the robustness of change blindness as a phenomenon: change blindness is inducible in a wide variety of situations from strict laboratory (31) to naturalistic (1) and virtual (41) settings. As expected

216 given this robustness, pilot testing indicated that the experimental manipulations were

217 successful in a range of hardware and software environments and usage scenarios.

218 Furthermore, the online study was followed up by a lab-based replication using the same 219 software in controlled conditions.

220 For the lab cohort, the above sources of variation were eliminated or reduced substantially 221 (with the exception of the unsystematic variation in stimulus and mask durations caused by JavaScript's imprecise timing control). In the lab-based part of the study the task was presented on a $37.1 \mathrm{~cm} \times 33.3 \mathrm{~cm}$ inch Dell monitor with a resolution of $1280 \times 1024$, a refresh rate of $60 \mathrm{~Hz}$, and colour depth of 32 Bit. The participants completed the task seated at a comfortable distance from the monitor of approximately 57 centimetres. 


\section{Paradigm selection}

227 The flicker paradigm was selected because of its ease of implementation and its greater resilience to variations in screen size. This resilience is due to the particular method of transient suppression deployed in the flicker paradigm: in a flicker paradigm there are no change-specific transients since the transition (in the masked condition) is from initial stimulus, to mask, to alternate stimulus. Other methods, such as the mudsplash paradigm (12), rely

232 upon attracting attention away from change-specific transients by introducing more salient 233 transients elsewhere. While these other methods are likely to work given the robustness of 234 change blindness as a phenomenon, screen size variation among online participants means that the distance between any two points on the screen could not be guaranteed to produce equal visual space distances for all participants. Thus, relying upon location-based transientsuppression would introduce unnecessary variation between participants.

\section{Results}

\section{Descriptives and exclusions}

240 Experimental data consisted of 3035 trials (online $=1545$, lab $=1490$ ) from 42 participants

241 (online $=27$, lab $=15$ ). Trials were excluded if the wrong object was identified as having 242 changed, if the trial took longer than 20 s, or if the trial belonged to a participant who either had 243 zero valid trials for any of the eight trial types or had an overall accuracy below $90 \%$ (Table 1).

244 Table 1. Trial exclusion process

\section{Criterion}

(sequentially applied)

All trials

Errors

$\mathrm{RT}>20$ s
Online

2226

120

139
Trial count

Lab

1746

229

81

109

220

\section{Running total}

3972 


$\begin{array}{lrrrr}\text { Missing trial types } & 190 & 0 & 190 & \mathbf{3 3 3 3} \\ \text { Accuracy }<90 \% & 232 & 66 & 298 & \mathbf{3 0 3 5}\end{array}$

246 The excluded trials were examined for differences in manipulation using $\mathrm{X}^{2}$ tests with Yates' 247 continuity correction, conducted on those trials performed by the 42 participants included in 248 the experimental data. Errors occurred significantly more often than expected by chance in 249 masked than unmasked trials $\left(\mathrm{X}^{2}(1, N=3354)=30.0, p<.001\right)$, in high than low load trials $250\left(\mathrm{X}^{2}(1, N=3354)=29.5, p<.001\right)$, and in orientation than trajectory trials $\left(\mathrm{X}^{2}(1, N=3354)=\right.$ $2515.67, p=.017)$.

252 Online participants completed an average of $57.2( \pm S D 4.49)$ trials, and lab participants 99.3 $253( \pm S D 5.83)$. There was no significant difference in the number of orientation and trajectory 254 trials included in the final statistical analysis for either the online $\left(t(214.0)=-0.227, M_{\text {diff }}=\right.$ 255 $0.139[95 \% \mathrm{Cl}:-1.35,1.07], p=.821)$ or lab cohort $\left(t(118.0)=-0.624, M_{\text {diff }}=-0.667[95 \% \mathrm{Cl}:-\right.$ 256 $2.78,1.45], p=.534)$. The distribution of trials by contingency for each cohort is shown in Error! Reference source not found.. These data, including excluded trials and participants, 258 have been made available along with the script used to analyse them (https://doi.org/ 259 10.6084/m9.figshare.6580223).

260 Fig 2. Trial type distribution. The trial type was selected at random at the beginning of each 261 trial. Boxplots show the distribution of the number of trials of each trial type completed 262 (successfully) by participants in the online and lab cohorts.

Analyses were performed with trials collapsed by participant. The means number of trials and response time for each condition, and their 95\% confidence intervals, are shown in Table 2. Response times are calculated from the moment responding is enabled (after the altered stimulus becomes visible) until a response is recorded. For masked trials, in which repetitions of the mask make responding more difficult, response times are reduced by the duration of 
269 the masks displayed. Analysis using unadjusted response times made no difference to the 270 pattern of results obtained.

271 Table 2. Means

Trial Type

Low load Masked Trajectory

Low load Unmasked Orientation

High load Masked Orientation

High load Unmasked Trajectory

Low load Masked Orientation

Low load Unmasked Trajectory

High load Masked Trajectory

\section{Number of Trials Response Time $(m s)$}

mean $\quad 95 \% \mathrm{Cl}$ mean $95 \% \mathrm{Cl}$

Online $\quad 11.2 \quad 9.4,12.9 \quad 1738.0 \quad 1105.9,2370.1$

Lab $\quad 18.5 \quad 16.4,20.6 \quad 1574.4 \quad 1195.4,1953.4$

Online $\quad 4.3 \quad 3.5,5.2 \quad 889.0 \quad 577.9,1200.1$

Lab $\quad 7.5 \quad 6.4,8.5 \quad 762.9 \quad 637.1,888.8$

Online $\quad 4.6 \quad 3.6,5.6 \quad 813.3 \quad 638.4,988.1$

Lab $\quad 9.5 \quad 8.0,10.9 \quad 722.4 \quad 604.8,840.1$

Online $\quad 8.1 \quad 6.3,9.9 \quad 3786.4 \quad 3012.1,4560.6$

Lab $\quad 14.4 \quad 12.1,16.7 \quad 4224.1 \quad 3597.0,4851.3$

Online $\quad 11.0 \quad 9.2,12.8 \quad 1282.7 \quad 942.7,1622.7$

Lab $\quad 18.6 \quad 16.7,20.5 \quad 754.0 \quad 651.9,856.0$

Online $\quad 4.5 \quad 3.5,5.4 \quad 1207.4 \quad 735.9,1678.9$

Lab $\quad 5.9 \quad 4.8,7.0 \quad 1034.7 \quad 814.4,1255.0$

Online $\quad 4.6 \quad 3.6,5.6 \quad 655.3 \quad 498.7,811.9$

Lab $\quad 8.5 \quad 6.6,10.5 \quad 486.7 \quad 449.6,523.8$

Online $\quad 9.0 \quad 7.3,10.6 \quad 3699.7 \quad 3002.1,4397.3$

Lab $\quad 16.4 \quad 14.0,18.8 \quad 3565.5 \quad 3077.4,4053.6$

274 Analysis of data from the lab cohort was preregistered (https://aspredicted.org/rh3d2.pdf) to

275 use a $2 \times 2 \times 2$ within-subjects ANOVA, and to be repeated covering both raw and adjusted

276 response time as a dependant variable. These analyses were conducted, but, given the 
277 similarity to the main analysis reported below, are not reported in detail here. Detailed results

278 from all analyses conducted in this paper, the raw data upon which they are based, and the 279 scripts used to produce them, are available online 280 https://doi.org/10.6084/m9.figshare.6580223.

The data were analysed with a mixed $(2 \times 2 \times 2 \times 2)$ ANOVA. Change type (orientation vs trajectory), masking (unmasked vs masked), and load (low vs high) were the within-subjects variables, and cohort (online vs lab) was the between-subjects variable. Main effects were observed for all three within-subjects factors: responses were slower when masked $(F(1,40)$ $=218.0, p<.001, \eta_{p}^{2}=.846, M_{\text {diff }}=-1358.7$ [95\%Cl: $\left.\left.-1677.7,-1039.7\right]\right) ;$ under high load $\left(F(1,40)=252.5, p<.001, \eta_{p}^{2}=.863, M_{\text {diff }}=-1750.6[95 \% \mathrm{Cl}:-2046.7,-1454.4]\right) ;$ and for orientation changes $\left(F(1,40)=14.3, p<.001, \eta_{p}^{2}=.285, M_{\text {diff }}=341.0[95 \% \mathrm{Cl}:-689.6,7.5]\right)$. A significant interaction was observed for masking $\mathrm{x}$ load, with the increase in response time for masked trials being exacerbated under high load $\left(F(1,40)=179.1, p<.001, \eta_{p}^{2}=.820\right)$, while other interactions were not significant (all $F(1,40)<1.66$, all $\left.p>.206, \eta_{p}^{2}<.085\right)$. There was no effect of the between subjects factor, either as a main effect $(F(1,40)=0.232, p=.633$, $\left.\eta_{p}^{2}=.006, M_{\text {diff }}=118.4[95 \% \mathrm{Cl}:-231.6,468.3]\right)$, or in interactions (all $F(1,40)<2.50$, all $p>$ .122 , all $\left.\eta_{p}^{2}<.059\right)$.

The presence of an interaction between masking and load (Fig 3) indicates that change blindness occurred. The absence of three-way interaction between that interaction and change type is consistent with the suggestion that the change blindness effect is equivalent between change types. These data are consistent with the hypothesis that trajectory changes are detected and missed in a similar manner to orientation changes.

300 Fig 3. Change blindness interaction. The key pattern of interaction, whereby the response time for high load masked trials is far greater than either masked or high load trials alone, is 
evident for both orientation and trajectory changes. This relationship is stable between the online and lab cohorts. Error bars show 95\% confidence intervals.

305 This analysis was checked for robustness under various different assumptions: using non306 adjusted response times; excluding trials with a response time under $200 \mathrm{~ms}(N=18)$; including 307 all trials with a non-erroneous response time; and analysing the cohorts separately. None of these alternate analyses resulted in a different core pattern of results (main effects of masking and load, and an interaction between them), though the main effect of change type was nonsignificant in some cases. For the lab cohort alone, a significant interaction arose between change type and load $\left(\eta_{p}^{2}=.414\right)$.

312 Finally, a reviewer noted that the dramatic disruption arising from refreshing the display after

313 the A' panel has finished (Fig 1b) constituted a larger disruption than the visual mask, and that 314 results may be artificially elongated by this effect. Since this only occurs on trials where 315 answers are not given within the first A' panel (i.e. RT $<=700 \mathrm{~ms}$ ), we also performed analysis in which response time was replaced as the dependant variable by a binary variable indicating 317 whether or not a response was made within the first A' panel. The mean is thus the proportion 318 of trial which were solved immediately, and is conceptually similar to measuring whether or 319 not the participant detected the change in a one-shot paradigm.

The core result was robust to this analysis: unmasked changes were more likely to be noticed immediately than masked changes $\left(F(1,40)=200.8, p<.001, \eta_{p}^{2}=.842, M_{\text {diff }}=.302[95 \% \mathrm{Cl}\right.$ : $.228, .377])$; changes were more likely to be noticed immediately under low load $(F(1,40)=$ 195.5, $\left.p<.001, \eta_{p}^{2}=.830, M_{\text {diff }}=.361[95 \% \mathrm{Cl}: .289, .432]\right) ;$ and these effects interacted with one another $\left(F(1,40)=28.1, p<.001, \eta_{p}^{2}=.414\right)$. Additionally, a main effect of change type was observed, with trajectory changes being noticed immediately more frequently than orientation changes $\left(F(1,40)=63.9, p<.001, \eta_{p}^{2}=.670, M_{\text {diff }}=.134[95 \% \mathrm{Cl}: .054, .213]\right)$. This effect of change type also interacted with the masking $x$ load interaction, resulting in a three- 
way interaction $\left(F(1,40)=9.58, p=.004, \eta_{p}^{2}=.229\right)$. The main effects of masking and change type interacted with cohort, both being increased by lab conditions (masking: $F(1,40)=11.7$, $p=.001, \eta_{p}^{2}=.226 ;$ change type: $\left.F(1,40)=17.3, p<.001, \eta_{p}^{2}=.302\right)$.

\section{Discussion}

The results demonstrate that change blindness was achieved using the implementation.

Where scenes were sparsely populated enough for the relevant properties of all objects to be

maintained in working memory, or where transients accompanying key changes were available, changes were noticed rapidly. Where working memory exhaustion and transient masking occurred simultaneously, changes were noticed far more slowly. This pattern of results is typical of change blindness in both direction and magnitude $(2,13,27,42)$.

The use of dynamic stimuli did not compromise the orientation change blindness effects, consistent with suggestions of $(26,43)$. The replication of change blindness to orientation changes validates the methodology used here, and the similar patterns of results in the

341 orientation and trajectory conditions suggests that change blindness was also evoked for trajectory changes. The existence of change blindness to trajectory changes implies that trajectory changes are capable of directing attention exogenously since change blindness involves a loss of exogenous attention manipulation when its triggers are suppressed.

\section{Investigation of trajectory change blindness}

346 Transients are not required to detect all changes, as occasional success in masked change

347 detection tasks proves (44), and thus there is a question as to whether there are classes of 348 short-term changes which are routinely detected without recourse to transients. The present study examines whether trajectory changes are a class of discriminable changes which do not depend upon the detection of transients or differences in patterns of transients.

351 Were trajectory change detection typically transient-dependant it would be expected that

352 trajectory change detection response time would be modified in a similar way to orientation 
change detection response time under change blindness manipulations. This is demonstrated statistically by the absence of an interaction between the type of change variable and the load and masking variables. While certain choices in the statistical analysis did indicate differences in the magnitude of the change blindness interaction between orientation and trajectory changes, the larger effects were for the trajectory trials and the interaction remained strong for both change types. Since trajectory change detection responded in the same way as orientation change detection, and since orientation change detection is driven by the detection of transients whose presence the experiment directly manipulated, the conclusion is invited that trajectory change detection relies on the detection of visual transients (or patterns of transients) in the same way that orientation change detection does.

Orientation and direction-of-motion are neurophysiologically related under some conditions since the direction of slow movement is computed by direction-of-motion cells and rapid movement is computed by a combination of direction-of-motion and orientation-sensitive cells $(45,46)$. Rapid motion was not a focus in the current design because the stimuli moved too slowly to produce motion streaks, but it is plausible that trajectory changes would be detected by the orientation of motion streaks in a rapid motion version of the current paradigm. In the study presented here it is likely that orientation and trajectory changes were detected by different neural populations: direction-of-motion cells for trajectory changes and orientationsensitive cells for orientation changes.

The trajectory-orientation differences were stable across other manipulations, but were pronounced enough to produce a significant main effect of change type under some statistical choices. The difference may be explained by the consequences of the changes. Orientation changes happen 'all at once' in the sense that the orientation after the change is as different

376 from the pre-change orientation as it will get. Trajectory changes are instantiated just as 377 quickly when the direction of motion is altered, but the spatial position of the object differs 378 increasingly from the extrapolated location along the original trajectory as time goes on. For changes which are not noticed immediately (due to transients orienting attention to the 
change), comparisons between incoming sensory data and an estimate derived by extrapolating from memory become more extreme over time for trajectory changes. This could result in trajectory changes being detected more quickly on average because the change is, in some sense, continuing to occur.

384 It is important to note here that the saliency of the trajectories was controlled through the use of objects moving on a two-dimensional plane and through randomisation of both initial trajectories and direction of deflection. It would be expected that in an experiment where changes increased the saliency of trajectories (e.g. to move towards the participant), those changes would be detected rapidly even where the change was masked. This expectation would be in keeping with previous findings suggesting a link between saliency and change blindness attenuation across various different kinds of saliency measures $(2,18,19)$.

\section{Conclusion}

This experiment replicates change blindness using a dynamic version of the flicker paradigm and shows that a pattern of results typical of change blindness can be obtained for trajectory changes.

The experiment demonstrates that detection of trajectory changes can be subject to change blindness. Change blindness is theorised to occur on the basis of the detection of transients, and thus this experiment can be taken to show that trajectory change detection depends on the detection of the fluctuations in the patterns of transients accompanying trajectory change. While there may be discriminations which can only be made using top-down mechanisms, the presence of transients driving a trajectory change suggests that bottom-up processes can account for, at the least, some discriminations regarding changes in higher-order object properties.

403 The differences between detection speed for orientation and trajectory changes suggest that 404 trajectory changes are detected more readily and are slightly more resistant to masking by 405 flicker. This may be due to the additional temporal information that expected trajectories afford. 
406 Alternatively, trajectories of the separate elements may be represented in a gist-like pattern of

407 movement which boosts the salience of a single trajectory deviation, whereas the orientation

408 alterations may require serial search for identification of change. Future research examining

409 eye movements during the detection of orientation and trajectory changes could further our

410 understanding of this difference.

\section{Acknowledgements}

412 Thanks are due to Alex Martin for vital feedback during the development of the testing 413 application, for assistance in locating participants, and for helpful comments on various drafts 414 of this manuscript. This work would have remained unpublished but for the insightful 415 comments and guidance from Paul Graham. Thanks also to Jenny Bosten for particularly 416 helpful comments and critique. Finally, the authors are grateful for the valuable contribution of 417 three reviewers, which resulted in a clearer and better manuscript.

\section{Author Contributions}

MJ designed the experiment, developed the application, analysed the data, and wrote the manuscript, all under the supervision of RC. NA conducted the lab-based replication and contributed to the drafting of the manuscript.

\section{References}

423

424

425

426

427

428

429

430

431
1. Simons DJ, Levin DT. Failure to detect changes to people during a real-world interaction. Psychon Bull Rev. 1998 Dec;5(4):644-9.

2. Rensink RA, O'Regan JK, Clark JJ. To See or not to See: The Need for Attention to Perceive Changes in Scenes. Psychol Sci. 1997 Sep 1;8(5):368-73.

3. Hughes HC, Caplovitz GP, Loucks RA, Fendrich R. Attentive and Pre-Attentive Processes in Change Detection and Identification. PLOS ONE. 2012 Aug 16;7(8):e42851.

4. Lyyra P, Mäkelä H, Hietanen JK, Astikainen P. Implicit Binding of Facial Features During Change Blindness. PLOS ONE. 2014 Jan 30;9(1):e87682. 
5. Gaspar JG, Neider MB, Simons DJ, McCarley JS, Kramer AF. Change Detection: Training and Transfer. PLOS ONE. 2013 Jun 28;8(6):e67781.

6. Borowsky A, Horrey WJ, Liang Y, Garabet A, Simmons L, Fisher DL. The effects of brief visual interruption tasks on drivers' ability to resume their visual search for a pre-cued hazard. Accid Anal Prev. 2016 Aug 1;93:207-16.

7. Charlton SG, Starkey NJ. Driving without awareness: The effects of practice and automaticity on attention and driving. Transp Res Part F Traffic Psychol Behav. 2011 Nov 1;14(6):456-71.

8. Lee Y-C, Lee JD, Ng Boyle L. Visual Attention in Driving: The Effects of Cognitive Load and Visual Disruption. Hum Factors J Hum Factors Ergon Soc. 2007 Aug;49(4):721-33.

9. Simons DJ, Ambinder MS. Change Blindness Theory and Consequences. Curr Dir Psychol Sci. 2005 Feb 1;14(1):44-8.

10. Mitroff SR, Simons DJ, Levin DT. Nothing compares 2 views: Change blindness can occur despite preserved access to the changed information. Percept Psychophys. 2004 Nov;66(8):1268-81.

11. Jensen MS, Yao R, Street WN, Simons DJ. Change blindness and inattentional blindness: Change blindness and inattentional blindness. Wiley Interdiscip Rev Cogn Sci. 2011 Sep;2(5):529-46.

12. O'Regan JK, Rensink RA, Clark JJ. Change-blindness as a result of 'mudsplashes'. Nature. 1999 Mar 4;398(6722):34-34.

13. Levin DT, Simons DJ. Failure to detect changes to attended objects in motion pictures. Psychon Bull Rev. 1997 Dec;4(4):501-6.

14. O'Regan JK, Deubel H, Clark JJ, Rensink RA. Picture Changes During Blinks: Looking Without Seeing and Seeing Without Looking. Vis Cogn. 2000 Jan 1;7(1-3):191-211.

15. Baddeley A. Working memory: looking back and looking forward. Nat Rev Neurosci. 2003 Oct;4(10):829-39.

16. Smith $\mathrm{H}$, Milne $\mathrm{E}$. Reduced change blindness suggests enhanced attention to detail in individuals with autism. J Child Psychol Psychiatry. 2009 Mar 1;50(3):300-6.

17. Chen W, Liu CH, Nakabayashi K. Beauty Hinders Attention Switch in Change Detection: The Role of Facial Attractiveness and Distinctiveness. PLOS ONE. 2012 Feb 29;7(2):e32897.

18. Ro T, Russell C, Lavie N. Changing Faces: A Detection Advantage in the Flicker Paradigm. Psychol Sci. 2001 Jan 1;12(1):94-9.

19. Jones BT, Jones BC, Smith $\mathrm{H}$, Copley $\mathrm{N}$. A flicker paradigm for inducing change blindness reveals alcohol and cannabis information processing biases in social users. Addiction. 2003 Feb 1;98(2):235-44.

20. White CB, Caird JK. The blind date: The effects of change blindness, passenger conversation and gender on looked-but-failed-to-see (LBFTS) errors. Accid Anal Prev. 2010 Nov 1;42(6):1822-30. 
21. Harms IM, Brookhuis KA. Dynamic traffic management on a familiar road: Failing to detect changes in variable speed limits. Transp Res Part F Traffic Psychol Behav. 2016 Apr 1;38:37-46.

22. Levin DT, Varakin DA. No pause for a brief disruption: Failures of visual awareness during ongoing events. Conscious Cogn. 2004 Jun 1;13(2):363-72.

23. Wallis G, Bulthoff $\mathrm{H}$. What's Scene and Not Seen: Influences of Movement and Task Upon What We See. Vis Cogn. 2000 Jan 1;7(1-3):175-90.

24. Vachon $F$, Vallières $B$, Jones $D$, Tremblay $S$. Nonexplicit Change Detection in Complex Dynamic Settings: What Eye Movements Reveal. Hum Factors. 2012 Dec 1;54:9961007.

25. Smith TJ, Lamont $P$, Henderson JM. Change Blindness in a Dynamic Scene Due to Endogenous Override of Exogenous Attentional Cues. Perception. 2013 Aug 1;42(8):884-6.

26. Cavanaugh J, Wurtz R. Change blindness for motion in macaque monkey. J Vis. 2002 Nov 15;2(7):16-16.

27. Hewlett $P$, Oezbek C. How Stimulus Variables Combine to Affect Change Blindness. Curr Psychol. 2012 Nov 8;31(4):337-48.

28. Pylyshyn ZW, Storm RW. Tracking multiple independent targets: Evidence for a parallel tracking mechanism*. Spat Vis. 1988 Jan 1;3(3):179-97.

29. Pylyshyn Z. Some puzzling findings in multiple object tracking: I. Tracking without keeping track of object identities. Vis Cogn. 2004 Oct 1;11(7):801-22.

30. Makovski T, Jiang YV. Feature binding in attentive tracking of distinct objects. Vis Cogn. 2009 Jan 1;17(1-2):180-94.

31. Bahrami B. Object property encoding and change blindness in multiple object tracking. Vis Cogn. 2003 Nov 1;10(8):949-63.

32. Scholl BJ, Pylyshyn ZW, Franconeri SL. When are featural and spatiotemporal properties encoded as a result of attentional allocation? In: Investigative Ophthalmology \& Visual Science. ASSOC RESEARCH VISION OPHTHALMOLOGY INC 9650 ROCKVILLE PIKE, BETHESDA, MD 20814-3998 USA; 1999. p. S797-S797.

33. Pylyshyn ZW, Annan V. Dynamics of target selection in Multiple Object Tracking (MOT). Spat Vis. 2006 Nov 1;19(6):485-504.

34. Crafty - JavaScript Game Engine, HTML5 Game Engine [Internet]. 2016 [cited 2016 Jan 1]. Available from: http://craftyjs.com/

35. R Core Team. R: A Language and Environment for Statistical Computing [Internet]. Vienna, Austria: R Foundation for Statistical Computing; 2017. Available from: https://www.R-project.org/

36. Wickham H. tidyverse: Easily Install and Load the 'Tidyverse' [Internet]. 2017. Available from: https://CRAN.R-project.org/package=tidyverse 
37. Hewson C, Vogel C, Laurent D. Internet research methods [Internet]. Sage; 2015 [cited 2016 Jun 30]. Available from: https://books.google.co.uk/books?hl=en\&lr=\&id=w8mICwAAQBAJ\&oi=fnd\&pg=PP1\&ot $\mathrm{s}=0$ __WAYJg0p\&sig=OhhYyGlkYMlgbZ4DcgnUWsfcnlo

38. Reips U-D. The methodology of Internet-based experiments. Oxf Handb Internet Psychol. 2007;373-390.

39. Meyerson P, Tryon WW. Validating Internet research: A test of the psychometric equivalence of Internet and in-person samples. Behav Res Methods Instrum Comput. 2003;35(4):614-620.

40. HTML Standard [Internet]. WHATWG.org. 2017 [cited 2017 May 26]. Available from: https://html.spec.whatwg.org/multipage/webappapis.html\#timers

41. Vasser M, Kängsepp M, Aru J. Change Blindness in 3D Virtual Reality. ArXiv150805782 Cs Q-Bio [Internet]. 2015 Aug 24 [cited 2016 Mar 15]; Available from: http://arxiv.org/abs/1508.05782

42. Rensink RA. Visual Search for Change: A Probe into the Nature of Attentional Processing. Vis Cogn. 2000 Jan 1;7(1-3):345-76.

43. Rich A, Gillam B. Failure to detect changes in color for lines rotating in depth: the effects of grouping and type of color change. Vision Res. 2000 Jun;40(10-12):1377-84.

44. Simons DJ, Rensink RA. Change blindness: past, present, and future. Trends Cogn Sci. 2005 Jan;9(1):16-20.

45. Apthorp D, Schwarzkopf DS, Kaul C, Bahrami B, Alais D, Rees G. Direct evidence for encoding of motion streaks in human visual cortex. Proc R Soc B Biol Sci [Internet]. 2013

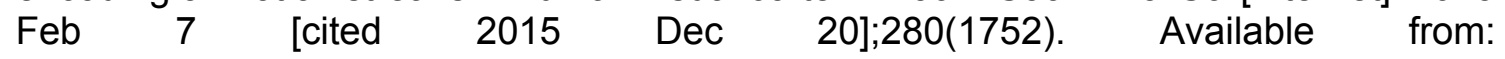
http://www.ncbi.nlm.nih.gov/pmc/articles/PMC3574303/

46. Geisler WS. Motion streaks provide a spatial code for motion direction. Nature. 1999 Jul 

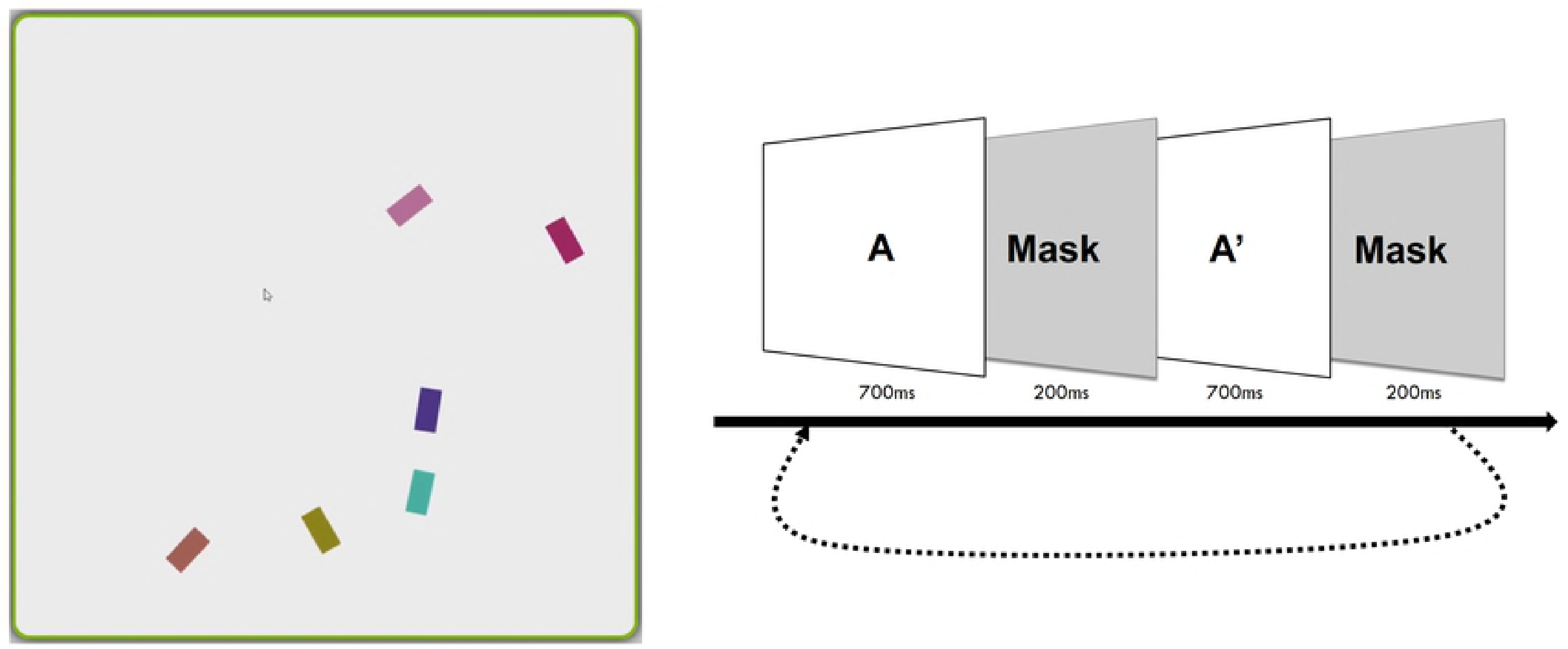
Online $\mathrm{n}=27$ 


\section{Unmasked $\multimap$ Masked}

bioRxiv preprint doi: https://doi.org/10.1101/391359; this version posted August 13, 2018. The copyright holder for this preprint (which was

not certified by peer review) is the author/funder, who has granted bioRxiv a license to display the preprint in perpetuity. It is made

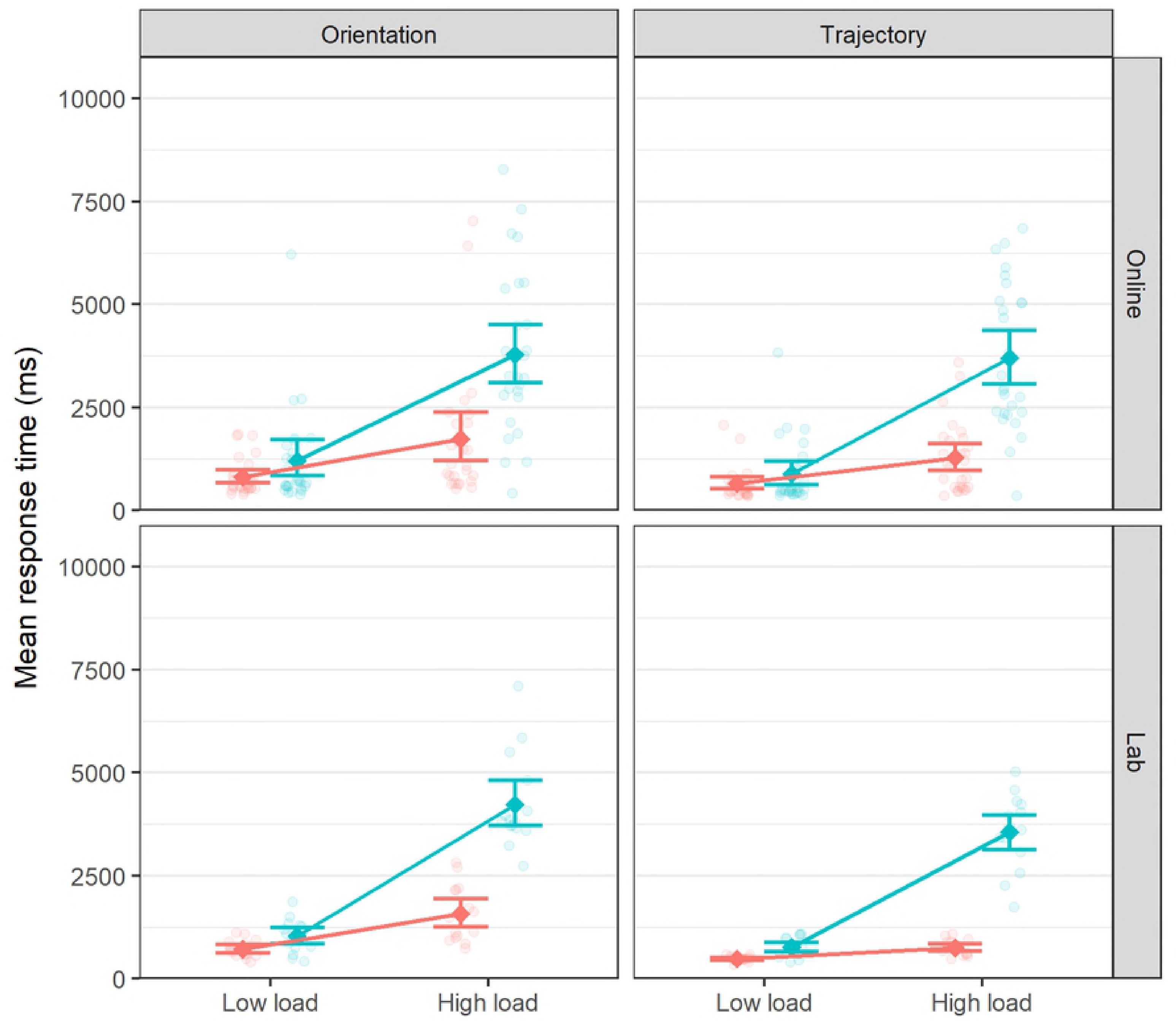

